\title{
Long-Chain Polyunsaturated Fatty Acids Modulate Lung Inflammatory Response Induced by Pseudomonas aeruginosa in Mice
}

\author{
STÉPHANE AUVIN, FRANÇOIS COLLET, FRÉDÉRIC GOTTRAND, MARIE-ODILE HUSSON, \\ XAVIER LEROY, CHRISTOPHER BEERMANN, AND BENOIT P. GUERY
}

\begin{abstract}
EA 2689 [S.A., F.C., B.P.G.], Faculté de Médecine de Lille, 59045 Lille, France; Department of Pediatrics [S.A., F.G.], Gastroenterology, Hepatology and Nutrition Unit, Hôpital Jeanne de Flandre, 59037 Lille, France; Department of Bacteriology [M.-O. H.], Department of Pathology [X.L.], Hôpital Calmette, 59037

Lille, France; Lipids research division [C.B.] Numico Research, D-61381 Friedrichsdorf, Germany
\end{abstract}

\begin{abstract}
Polyunsaturated fatty acid (PUFA) immunomodulatory properties have been studied extensively in chronic infections. Few studies have focused on acute infection; thus, PUFA effects in a mouse model of Pseudomonas aeruginosa (PA)-induced lung injury were evaluated. C57BL/6 mice were randomized to be fed for $3 \mathrm{wk}$ with an eicosapentaenoic acid (EPA) diet, an arachidonic acid (AA) diet, or a control diet [saturated fatty acids]. Lung injury was induced by intratracheal instillation of $10^{7} \mathrm{CFU}$ of PA per mouse. In each diet group, animals were studied either without or after PA-inducing lung injury. Evaluation criteria were early mortality; inflammatory response assessed with tumor necrosis factor- $\alpha$ (TNF- $\alpha)$, IL-1 $\beta$, IL-6 and IL-10 levels in bronchoalveolar lavage; lung injury evaluation; and extravascular lung water, assessed $24 \mathrm{~h}$ after the injury. After PA-induced lung injury, no difference in early mortality was observed; TNF- $\alpha$ level was significantly higher in the EPA diet than in the other two diet groups. No difference for the other cytokines was found among the groups. Lung edema was also more important in the EPA group, consistent with the variations of TNF- $\alpha$ levels.
\end{abstract}

\section{ABSTRACT}

Our study clearly shows that in PA-induced acute lung injury, n-3 PUFA induces differences in the inflammatory response with a higher level of lung edema. Modulation of the inflammatory response with n-3 PUFA can influence the response to a bacterial challenge. (Pediatr Res 58: 211-215, 2005)
AA, arachidonic acid
Abbreviations
AIN, American Institute of Nutrition
BAL, bronchoalveolar lavage
EPA, eicosapentaenoic acid
PA, Pseudomonas aeruginosa
PUFA, polyunsaturated fatty acid
$\mathbf{Q}_{\mathbf{B}}$, weight of intrapulmonary blood
$\mathbf{Q}_{\mathbf{D}}$, dry lung weight
$\mathbf{Q}_{\mathbf{W}}$, wet lung weight
SF, saturated fatty acids
TNF- $\alpha$, tumor necrosis factor- $\alpha$
W/D, wet to dry weight ratio

The effects of polyunsaturated fatty acids (PUFAs) in the modulation of the inflammatory response are now well established $(1,2)$. Different authors showed after an omega 3 fatty acid diet that isolated peripheral blood mononuclear cells had a decreased ex vivo production of tumor necrosis factor- $\alpha$ (TNF- $\alpha$ ), IL-1, and IL-6 (3-5). Several hypotheses have been raised to explain this phenomenon; the most interesting is based on the biochemical properties of eicosapentaenoic acid

Received November 11, 2004; accepted November 23, 2004.

Correspondence: Frédéric Gottrand, M.D., Ph.D., Unité de Gastro-entérologie, Hépatologie et Nutrition, Clinique de Pédiatrie, Hôpital Jeanne de Flandre, 2, avenue Oscar Lambret, 59037 Lille cedex, France; e-mail: fgottrand@chru-lille.fr.

EA 2689, Infectious Disease Research Laboratory, and a grant from Numico Research and Vaincre la mucoviscidose association supported this study.

DOI: 10.1203/01.PDR.0000169979.27641.40
(EPA). EPA can be converted to the 3 -series prostaglandins and 5-series leukotrienes (6); these mediators are considered less biologically active and less inflammatory than the arachidonic acid (AA)-derived 2-series prostaglandins and 4-series leukotrienes $(7,8)$. Lung functional consequences of PUFA supplementation have also been studied omega 3 fatty acid can reduce both lung membrane injury (9) and lung edema accumulation (10-12). As a consequence, an improvement of the survival rate has also been shown after infection caused by different microorganisms (13-15). These models used different types of bacteria, including Gram-negative bacilli; among them, Pseudomonas aeruginosa (PA) has never been studied.

$\mathrm{PA}$ is frequently encountered in intensive care units, being responsible for nosocomial pneumonia (16). This pathogen is associated with a high mortality rate, reaching $70 \%$ in some studies (17). Alteration in dietary lipid composition in the 
intensive care unit patients could be an attractive therapeutic target to modulate the host response.

The aim of this study was to determine the effect of PUFAs in a murine model of acute lung injury induced by PA. To evaluate the benefit of a preventive diet, we analyzed the inflammatory response at baseline and after the injury, as well as the alveolar capillary barrier permeability and lung edema.

\section{METHODS}

Animals. Male C57BL/6 mice (6 wk of age), purchased from CharlesRiver, were housed in the Lille University animal care facility and fed with three different diets produced by Numico Research (Friedrichsdorf, Germany). Food and water were available ad libitum. All mice were maintained in a protected unit. All experiments were performed with approval of the Lille institutional animal care and use committee.

Foods. The basis of our diets was adapted from the AIN-93 (American Institute of Nutrition) rodent diet (18). We used the rodent diet formulated for growth (AIN-93G; Table 1). We substituted AIN-93G lipids (soybean oil) by three different isocaloric fatty acid mixtures: EPA, AA, and saturated fatty acids (SF; Table 2).

Design of the study. Mice first were randomized in one of the three diet groups for 3 wk (Fig. 1). All experiments were performed blindly. A second randomization decided whether the animal would be infected with PA. Data analysis was performed $24 \mathrm{~h}$ after the instillation. Twenty mice were used in each group (10 for the inflammatory response and 10 for lung physiologic studies).

Experimental model of acute lung injury. PA (PAO1 strain) was incubated in $125 \mathrm{~mL}$ of tryptic soy broth at $37^{\circ} \mathrm{C}$ in a rotating shaking water bath for $8 \mathrm{~h}$. The culture then was washed twice with PBS and resuspended in PBS. The resulting bacterial suspension at $1 \times 10^{9} \mathrm{CFU} / \mathrm{mL}$ was diluted at 1:10 $\left(10^{8}\right.$ $\mathrm{CFU} / \mathrm{mL}$ ). Mice were anesthetized with sevoflurane (Servorane; Abbott, UK) and placed in dorsal recumbency. Acute lung injury was produced according to the method described by Pennington and Ehrie (19). Transtracheal insertion of a 24-G animal feeding needle was used to instill $100 \mu \mathrm{L}$ of the inoculum $\left(10^{7} \mathrm{CFU} /\right.$ mouse).

Evaluation of lung inflammatory response. Bronchoalveolar lavage (BAL) was performed by cannulating the trachea in situ with a 22-G, 1.5-inch bead-tipped feeding needle; four aliquots of $0.5 \mathrm{~mL}$ of sterile PBS were instilled; and the fluid was collected by aspiration. BAL samples were filtered and immediately frozen at $-80^{\circ} \mathrm{C}$ after collection. Murine TNF- $\alpha$, IL-1, IL-6, and IL-10 were measured using commercially available sandwich enzyme immunoassays (Quantikine; R\&D Systems, Abingdon OX, UK). BAL samples were assayed in duplicate and compared with known standards.

Evaluation of endothelial injury: measurement of the alveolar capillary membrane permeability. To evaluate the endothelial injury, we calculated the albumin flux across the endothelial barrier using a previously described method adapted from rats (20). Briefly, $2 \mathrm{~h}$ before the experimentation, $0.5 \mathrm{~mL}$ of ${ }^{125} \mathrm{I}$-labeled human serum albumin (HAS; $1 \mu \mathrm{Ci}$; CIS Biointernational, Gif sur Yvette, France) was injected intraperitoneally. After i.p. injection of pentobarbital sodium (Sanofi, Libourne, France), exsanguination was performed through the jugular vein. The lungs were removed through a sternotomy, and blood radioactivity and $\mathrm{Hb}$ were measured.

Lung weight and radioactivity count were measured before homogenization (polytron, PT 1600 E; Fisher Bioblock Scientific, Switzerland) and centrifugation. The supernatant $\mathrm{Hb}$ content was measured. Blood and lung homogenate samples were placed at $37^{\circ} \mathrm{C}$ during $7 \mathrm{~d}$ to determine the wet to dry weight ratio $(\mathrm{W} / \mathrm{D})$.

Table 1. Diet composition in gram per kilogram of diet

\begin{tabular}{lc}
\hline \multicolumn{1}{c}{ Ingredient } & AIN-93G $(\mathrm{g} / \mathrm{kg}$ diet $)$ \\
\hline Cornstarch & 397 \\
Casein & 200 \\
Dextrinized cornstarch & 132 \\
Sucrose & 100 \\
Soybean oil & 70 \\
Fiber & 50 \\
Mineral mix & 35 \\
Vitamin mix & 10 \\
L-cystine & 3 \\
Choline bitartrate & 2.5 \\
\hline
\end{tabular}

Table 2. Repartition of each fatty acid in the three diet groups (gram per 100 grams of lipids)

\begin{tabular}{lccc}
\hline \multicolumn{1}{c}{ Fatty acid } & EPA diet & AA diet & SF Diet \\
\hline C16:0 (PA) & 9.0 & 20.0 & 30.0 \\
C18:0 (SA) & 3.0 & 7.0 & 10.0 \\
Total of saturated FA & 12.0 & 27.0 & 40.0 \\
MUFA oleic acid & 45.0 & 45.0 & 33.0 \\
C18:2 $\omega 6$ (LA) & 23.0 & 23.0 & 27.0 \\
C18:3 $\omega 3$ (ALA) & 10.0 & 0.1 & 0.0 \\
C18:3 $\omega 6$ (GLA) & 3.0 & 0.1 & 0.0 \\
C18:4 $\omega 3$ (STA) & 2.0 & 0.1 & 0.0 \\
C20:4 $\omega 6$ (AA) & 0.5 & 5.0 & 0.0 \\
C20:5 $\omega 3$ (EPA) & 5.0 & 0.5 & 0.0 \\
C22:6 $\omega 3$ (DHA) & 2.0 & 0.2 & 0.0 \\
Total of PUFA & 43.0 & 28.0 & 27.0 \\
\hline
\end{tabular}

MUFA, mono-unsaturated fatty acid; PA, palmitic acid; SA, stearic acid; LA, linoleic acid; AA, arachidonic acid; ALA, $\alpha$-linolenic acid; GLA, $\gamma$-linolenic acid; STA, stearidonic acid; EPA, eicosapentaenoic acid; DHA, docosahexaenoic acid; PUFA, polyunsaturated fatty acid; SF, Control diet with saturated fatty acids.

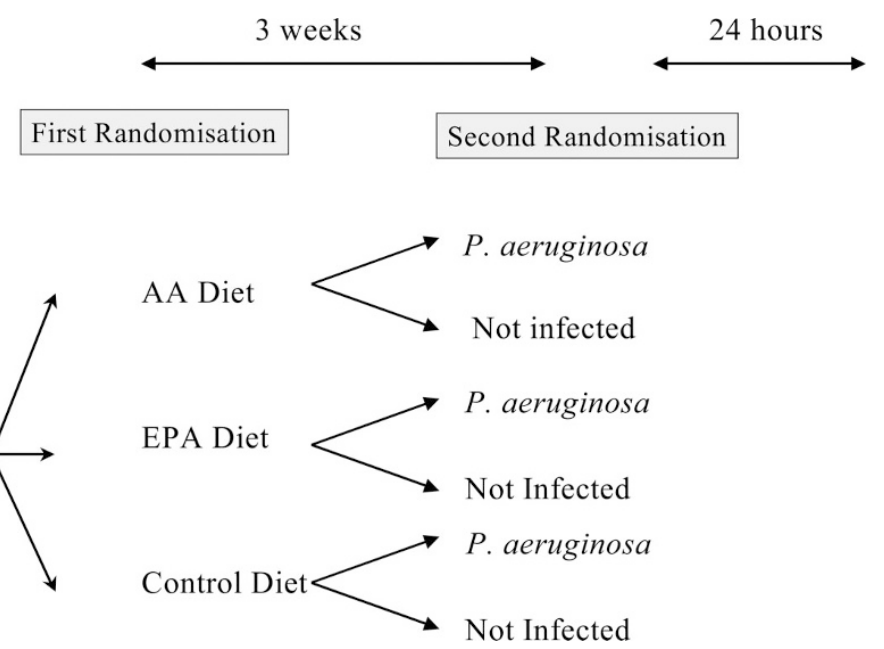

Figure 1. Design of the study.

\section{Permeability. We defined}

Extravascular lung water. Lung W/D was determined by removing the lung at the end of the experiment and recording the wet weight. The lung then was placed in a $37^{\circ} \mathrm{C}$ incubator for $7 \mathrm{~d}$, at which time the dry weight was recorded. For each pair of lungs, the W/D then was calculated.

Lung histopathology. Lungs were fixed in $2 \%$ paraformaldehyde for at least $48 \mathrm{~h}$, then dissected midsagittally and embedded in paraffin. Serial lung sections $(5 \mu \mathrm{m}$ thick) were stained with hematoxylin-eosin using standard techniques and examined for histopathologic changes. Diffuse alveolar damage was evaluated using a blind histologic scoring technique; three animals were analyzed in each group. Lung samples were divided into 20 observation fields at $\times 40$ magnification and graded on a scale of 0 to $3(0=$ absent, $1=$ mild, $2=$ moderate, and $3=$ intense) for inflammation. The lung injury was described as focal or diffuse for each sample.

Statistical Analysis. Data are expressed as the mean \pm SEM. KruskallWallis one-way ANOVA using Dunn's method to compare differences between groups and Mann-Whitney rank sum test analysis were performed using SPSS (11.0.1 version; LEAD Technologies, Chicago, IL). Statistical significance was considered at $p \leq 0.05$.

\section{RESULTS}

Basal inflammatory response was comparable between groups. After 3 wk of diet before PA acute infection, all groups were comparable for all of the studied variables. There was no significant difference among the three diet groups regarding cytokine levels, lung permeability, and W/D (Table 3). 
Table 3. Secretion of cytokines and physiological parameters in the different diet groups without Pseudomonas aeruginosa infection

\begin{tabular}{|c|c|c|c|c|}
\hline & $\begin{array}{l}\text { EPA Diet } \\
(n=10)\end{array}$ & $\begin{array}{l}\text { AA Diet } \\
(n=10)\end{array}$ & $\begin{array}{c}\text { SF Diet } \\
(n=10)\end{array}$ & $p$ \\
\hline $\operatorname{IL} 1 \beta(\mathrm{pg} / \mathrm{ml})$ in BAL & 0 & 0 & 0 & - \\
\hline IL6 $(\mathrm{pg} / \mathrm{ml})$ in BAL & 0 & 0 & 0 & - \\
\hline $\mathrm{TNF} \alpha(\mathrm{pg} / \mathrm{ml})$ in BAL & 0 & 0 & 0 & - \\
\hline Indexed Permability & $0.73 \pm 0.23$ & $0.84 \pm 0.28$ & $0.84 \pm 0.46$ & 0.77 \\
\hline $\mathrm{W} / \mathrm{D}$ & $4.71 \pm 0.36$ & $4.64 \pm 1.06$ & $4.74 \pm 0.51$ & 0.95 \\
\hline
\end{tabular}

IL, interleukin; BAL, Broncho-alveolar liquid; I Perm, indexed permeability; W/D, wet to dry weight lung ratio. Results are expressed as means and standard error of the mean.

$P A$ instillation significantly increased cytokine levels in the $\boldsymbol{B A L}$. Independent of the diet group, there was a significant difference for all cytokine measurements between controls and PA-infected animals. Alveolar capillary membrane permeability also increased in infected groups (Fig. 2). Mortality rates were 20, 27, and $13 \%$ for the EPA, AA, and SF diet groups, respectively (NS).

EPA increased TNF production and W/D after PA infection. TNF- $\alpha$ levels in the BAL were, respectively, 1752, 1171, and $578 \mathrm{pg} / \mathrm{mL}$ for the EPA, AA, and SF diets ( $p=0.01$; Fig. 3 ). Mann-Whitney rank sum test analysis found a higher level of TNF- $\alpha$ in the EPA diet group compared with the other groups. Consistent with an increased injury, extravascular lung water significantly increased in the EPA group. However, the permeability was not different among the three groups (Figs. 3 and 4).

No difference was observed in histologic feature between groups. Histologic analysis showed an interstitial infiltration with polymorphonuclear cells, associated with blood vessel congestion and bronchial lysis in all groups after PA infection. No difference, based on the histologic scoring, was observed among the different diets (Table 4).

\section{DISCUSSION}

A 3-wk dietary supplementation with various PUFAs before PA lung injury influences the acute inflammatory response and lung functional consequences demonstrated by the accumulation of extravascular water. Using a sublethal bacterial load, the aim of the study was to evaluate the immunomodulatory effect of PUFAs in the early response in severe acute lung injury.

Immunomodulatory effects of PUFA. PUFAs have immunomodulatory properties that previously have been described:

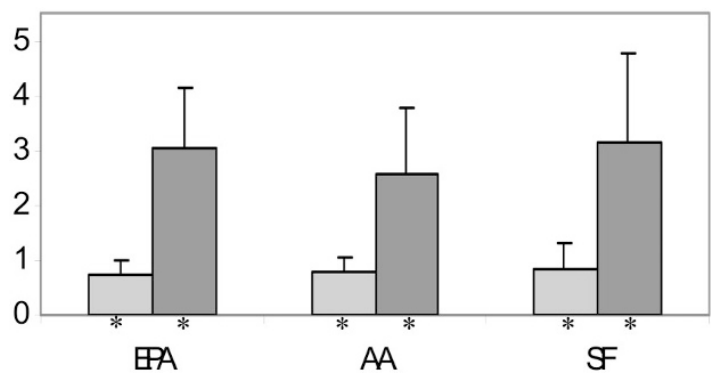

Figure 2. Indexed permeability in infected $(n=10)$ and noninfected $(n=10)$ animals. Results are expressed as means and SEM. $\square$ Noninfected; $\square$ Infected. $* p<0.0001 v s$ the infected groups. by down-regulating the inflammatory response, omega $3 \mathrm{PU}$ FAs can be opposed to omega 6 PUFAs, which up-regulate these parameters $(1,2)$. Endres et al. (3) studied the influence of $\mathrm{n}-3$ fatty acid dietary supplementation in healthy volunteers. In vitro, endotoxin-stimulated peripheral blood mononuclear cells presented a $43 \%$ decrease of IL- $1 \beta$ release after $6 \mathrm{wk}$ of supplementation; an additional $18 \%$ decrease was observed 10 wk after the end of the supplementation. The production of IL- $1 \alpha$ and TNF- $\alpha$ followed the same pattern. Twenty weeks after the end of the supplementation, the production of IL- $1 \beta$, IL- $1 \alpha$, and TNF- $\alpha$ returned to the presupplemental level. The decreased production of IL-1 and TNF- $\alpha$ was associated with a decreased ratio of AA to EPA in mononuclear cell membrane phospholipids. In a comparable work, Meydani et al. (4) showed after 3 mo that n-3 fatty acid supplementation reduced total IL-1 $\beta$, TNF- $\alpha$, and IL-6.

In our study, the diets' compositions were designed to provide similar quantities of essential fatty acids but predominance in one PUFA series (Table 2) to appreciate the effect of each fatty acid series while in most of the other studies, PUFA effects are usually analyzed by changing the omega- 6 to omega-3 ratio. We found a surprisingly higher TNF- $\alpha$ level in BAL in the EPA diet group compared with the other groups, conflicting with several other studies. This higher level of TNF- $\alpha$ was not associated with any difference in bacterial clearance from the lungs (data not shown). These discrepancies could be related to the injury itself and the inflammatory response generated after PA instillation; in fact, this bacterium is particularly pathogenic with a lot of different virulence mechanisms. Moreover, it has been reported that species differences after lipid diets and the increase that we observed are consistent with other published work in mice $(21,22)$.

PUFA effects on lung permeability. In PA-induced acute lung injury as in our study, alveolar capillary permeability is increased on both epithelial and endothelial barriers $(23,24)$. Using different fat blends, Mancuso et al. (9) studied the influence of PUFAs on alveolar capillary permeability in a Salmonella enteritidis endotoxin model. In this study, alveolar protein permeability increased and hypotension was reduced with PUFA fat blends that contained more omega 3 fatty acids. To our knowledge, no other study has assessed PUFA effect on lung permeability. In our study, we failed to find any difference in permeability between groups. This could be related to the lack of sensitivity of the methods used to study lung permeability in mice compared with rats. 

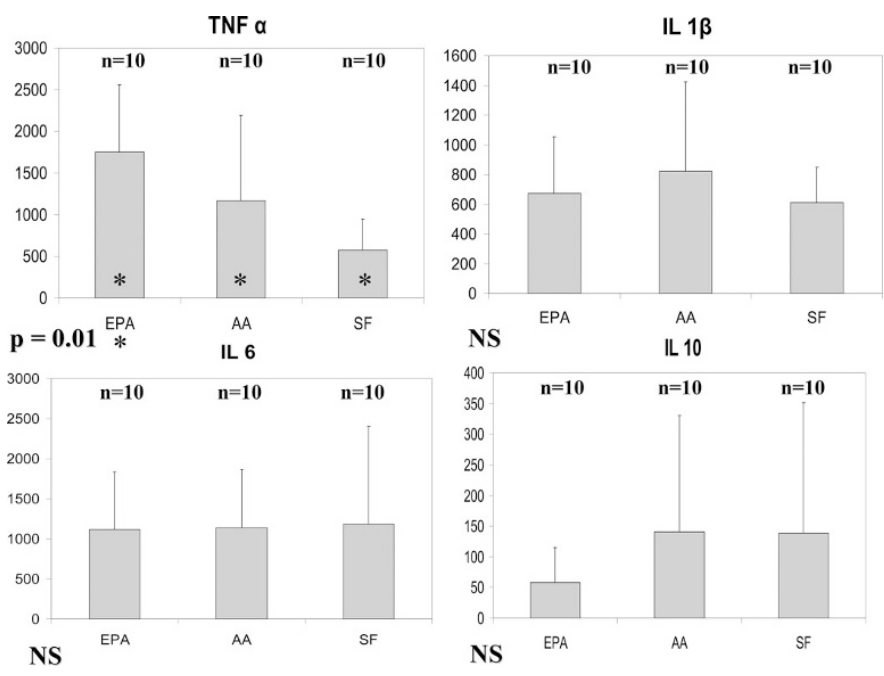

Figure 3. Measurement of cytokines in the BAL in PA-injured mice. Results are expressed as means and SEM. ${ }^{*} p<0.05 v s$ the two other groups.

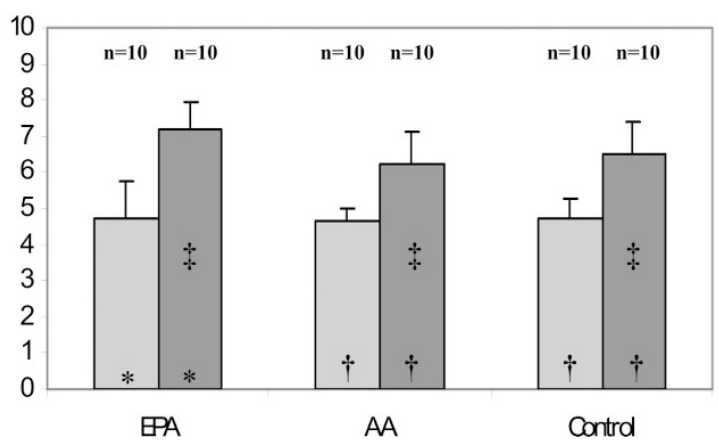

Figure 4. Lung W/D in infected and noninfected animals. Results are expressed as means and SEM. $\square$ Noninfected; $\square$ Infected. ${ }^{*} p=0.001 ; \dagger p=$ $0.0001 ; \ddagger p=0.03 ;{ }^{*} p<0.001$ vs the infected groups.

PUFA effects on lung edema. In an isolated perfused lung model, Koch et al. (12) demonstrated that omega-3 fatty acid infusion decreased edema formation after chemical injury. Breil et al. (10), in a comparable setting, showed that n-3 lipid infusion had anti-inflammatory effects on lung vasculature. These authors discussed a role of the EPA metabolism with a generation of less potent inflammatory eicosanoids resulting in a reduced edema. Sane et al. (11) reported, in a rat endotoxin model, that EPA supplementation depressed AA content and leukotriene B (4) generation in alveolar macrophages as well as plasma thromboxane B (2), leading to decreased pulmonary edema. Omega-3 fatty acids also reduce the release of proinflammatory lipid-derived mediators, consistent with a decrease of lung edema level.

In our study, we found a higher level of edema in the EPA diet group compared with the others. These discrepancies compared with the literature could also be related to the model that we used. In facts bacterial pneumonia induces a strong inflammatory response. The increase of the extravascular lung water that we found may reflect an alteration of the equilibrium between alveolar liquid clearance and pulmonary liquid clearance. A major inflammatory response may also be responsible for this increase; in this hypothesis, EPA could be responsible
Table 4. Histological scoring of PA injured lungs

\begin{tabular}{lccc}
\hline \multicolumn{1}{c}{ Diet group } & EPA & AA & SF \\
$(n=3)$ & $(n=3)$ & $(n=3)$ \\
\hline Degree of inflammation & 2.33 & 2.33 & 2.66 \\
Extension & $\mathrm{D}$ & $\mathrm{D}$ & $\mathrm{D}$ \\
\hline
\end{tabular}

AA, Arachidonic acid diet group; EPA, Eicosapentaenoic acid diet group; SF, control diet group. The scale was 0 to 3 (0: absent, 1: mild, 2: moderate, 3: intense) for inflammation. $\mathrm{D}$, diffuse.

for an enhancement of the animal inflammatory response, and the increased extravascular lung water could reflect a side effect, consistent with the higher levels of TNF- $\alpha$ in the EPA group.

Effect of PUFAs in mortality after bacterial infection. Previous reports showed that fish oil, containing high concentration of omega- 3 fatty acids, could prevent mortality in infectious models. This was documented in Klebsiella pneumoniae infection (14), Staphylococcus aureus and Bacteroides fragilis abscesses models (13), and Klebsiella pneumoniae and Plasmodium berghei infections (15). This parameter was never evaluated in PA acute lung injury. In our study, we did not find any difference in early mortality among the groups. However, our study was not designed to do so, because the animals were killed very early after infection $(24 \mathrm{~h})$. It therefore was not possible to assess the consequences on spontaneous mortality rate on long-term follow-up.

In conclusion, our study clearly shows that PUFAs can modulate the inflammatory response. After a PA challenge, we observed major changes in the early phase of the injury, depending on the diet of the animals, EPA being associated with an increase of TNF production as well as lung edema. On this basis, the question of whether this effect on inflammation could be beneficial for the animal should be studied with a long-term assessment of the inflammatory response and the outcome.

\section{REFERENCES}

1. Blok WL, Katan MB, van der Meer JW 1996 Modulation of inflammation and cytokine production by dietary (n-3) fatty acids. J Nutr 126:1515-1533

2. Grimble RF 1998 Nutritional modulation of cytokine biology. Nutrition 14:634-640

3. Endres S, Ghorbani R, Kelley VE, Georgilis K, Lonnemann G, van der Meer JW, Cannon JG, Rogers TS, Klempner MS, Weber PC 1989 The effect of dietary supplementation with n-3 polyunsaturated fatty acids on the synthesis of interleukin-1 and tumor necrosis factor by mononuclear cells. N Engl J Med 320:265-271

4. Meydani SN, Endres S, Woods MM, Goldin BR, Soo C, Morrill-Labrode A, Dinarello CA, Gorbach SL 1991 Oral (n-3) fatty acid supplementation suppresses cytokine production and lymphocyte proliferation: comparison between young and older women. J Nutr 121:547-555

5. Meydani SN, Lichtenstein AH, Cornwall S, Meydani M, Goldin BR, Rasmussen H, Dinarello CA, Schaefer EJ 1993 Immunologic effects of national cholesterol education panel step- 2 diets with and without fish-derived N-3 fatty acid enrichment. J Clin Invest 92:105-113

6. Lands WE 1992 Biochemistry and physiology of n-3 fatty acids. FASEB J 6:2530 2536

7. Lee TH, Menica-Huerta JM, Shih C, Corey EJ, Lewis RA, Austen KF 1984 Characterization and biologic properties of 5,12-dihydroxy derivatives of eicosapentaenoic acid, including leukotriene B5 and the double lipoxygenase product. J Biol Chem 259:2383-2389

8. Fischer S, Weber PC 1984 Prostaglandin I3 is formed in vivo in man after dietary eicosapentaenoic acid. Nature 307:165-168

9. Mancuso P, Whelan J, DeMichele SJ, Snider CC, Guszcza JA, Claycombe KJ, Smith GT, Gregory TJ, Karlstad MD 1997 Effects of eicosapentaenoic and gamma-linolenic acid on lung permeability and alveolar macrophage eicosanoid synthesis in endotoxic rats. Crit Care Med 25:523-532

10. Breil I, Koch T, Heller A, Schlotzer E, Grunert A, van Ackern K, Neuhof H 1996 Alteration of n-3 fatty acid composition in lung tissue after short-term infusion of fish oil emulsion attenuates inflammatory vascular reaction. Crit Care Med 24:1893-1902 
11. Sane S, Baba M, Kusano C, Shirao K, Andoh T, Kamada T, Aikou T 2000 Eicosapentaenoic acid reduces pulmonary edema in endotoxemic rats. J Surg Res 93:21-27

12. Koch T, Duncker HP, Klein A, Schlotzer E, Peskar BM, van Ackern K, Neuhof H 1993 Modulation of pulmonary vascular resistance and edema formation by shortterm infusion of a 10\% fish oil emulsion. Infusionsther Transfusionsmed 20:291-300

13. Barton RG, Wells CL, Carlson A, Singh R, Sullivan JJ, Cerra FB 1991 Dietary omega-3 fatty acids decrease mortality and Kupffer cell prostaglandin E2 production in a rat model of chronic sepsis. J Trauma 31:768-773

14. Bjornsson S, Hardardottir I, Gunnarsson E, Haraldsson A 1997 Dietary fish oil supplementation increases survival in mice following Klebsiella pneumoniae infection. Scand J Infect Dis 29:491-493

15. Blok WL, Vogels MT, Curfs JH, Eling WM, Buurman WA, van der Meer JW 1992 Dietary fish-oil supplementation in experimental gram-negative infection and in cerebral malaria in mice. J Infect Dis 165:898-903

16. George DL, Falk PS, Wunderink RG, Leeper KV Jr, Meduri GU, Steere EL, Corbett CE, Mayhall CG 1998 Epidemiology of ventilator-acquired pneumonia based on protected bronchoscopic sampling. Am J Respir Crit Care Med 158:1839-1847

17. Crouch Brewer S, Wunderink RG, Jones CB, Leeper KV Jr 1996 Ventilatorassociated pneumonia due to Pseudomonas aeruginosa. Chest 109:1019-1029
18. Reeves PG 1997 Components of the AIN-93 diets as improvements in the AIN-76A diet. J Nutr 127:838S-841S

19. Pennington JE, Ehrie MG 1978 Pathogenesis of Pseudomonas aeruginosa pneumonia during immunosuppression. J Infect Dis 137:764-774

20. Jayr C, Garat C, Meignan M, Pittet JF, Zelter M, Matthay MA 1994 Alveolar liquid and protein clearance in anesthetized ventilated rats. J Appl Physiol 76:2636-2642

21. Chavali SR, Weeks CE, Zhong WW, Forse RA 1998 Increased production of TNF-alpha and decreased levels of dienoic eicosanoids, IL-6 and IL-10 in mice fed menhaden oil and juniper oil diets in response to an intraperitoneal lethal dose of LPS. Prostaglandins Leukot Essent Fatty Acids 59:89-93

22. Petursdottir DH, Olafsdottir I, Hardardottir I 2002 Dietary fish oil increases tumor necrosis factor secretion but decreases interleukin-10 secretion by murine peritoneal macrophages. J Nutr 132:3740-3743

23. Kudoh I, Wiener-Kronish JP, Hashimoto S, Pittet JF, Frank D 1994 Exoproduct secretions of Pseudomonas aeruginosa strains influence severity of alveolar epithelial injury. Am J Physiol 267:L551-L556

24. Rezaiguia S, Garat C, Delclaux C, Meignan M, Fleury J, Legrand P, Matthay MA, Jayr C 1997 Acute bacterial pneumonia in rats increases alveolar epithelial fluid clearance by a tumor necrosis factor-alpha-dependent mechanism. J Clin Invest 99:325-335 